\title{
Performance Analysis of Relay Networks with Channel code in Low SNR Regime
}

\author{
Thang X. Vu ${ }^{\dagger}$, Quoc Bao Vo Nguyen*, Marco Di Renzo ${ }^{\dagger}$ and Pierre Duhamel ${ }^{\dagger}$ \\ ${ }^{\dagger}$ Laboratory of Signals and Systems (LSS), French National Center for Scientific Research (CNRS) - \\ École Supérieure d'Électricité (SUPÉLEC) - University of Paris-Sud 11, \\ 3 rue Joliot-Curie, 91192 Gif-sur-Yvette (Paris), France, \\ E-Mail: \{xuanthang.vu, marco.direnzo, pierre.duhamel\}@1ss.supelec.fr. \\ ${ }^{*}$ The Posts and Telecommunications Institute of Technology, Ho Chi Minh City, Vietnam \\ E-Mail: baovnq@ptithcm.edu.vn
}

\begin{abstract}
This paper analyzes the performance of relay networks with channel coding in low and medium Signal-to-Noise Ratio (SNR) regime. In particular, we study the three-node relay network in the quasi-static block Rayleigh fading channel plus additive white Gaussian noise. Estimate-and-forward (EF) relay protocol is used. In order to achieve high spectrum efficiency, the relay can either forward the whole or a part of the estimated codeword to the destination. The contributions of the paper are as follows: i) First, we compute an upper bound of the Bit Error Rate (BER) of the proposed scheme. ii) Second, from the upper bound, we derive a so-called instantaneous diversity order in low and medium SNR region which is essential to practical systems. The instantaneous diversity depends on both the amount of information forwarded by the relay and the minimum distance of the channel code. Interestingly, the proposed scheme can achieve full diversity gain in a given SNR region of interest (such as BER $\leq 10^{-5}$ ) while obtaining $32 \%$ spectrum efficiency improvement compared to classical relay network under appropriate conditions. The analysis is checked by simulation.
\end{abstract}

\section{INTRODUCTION}

Cooperation among nodes is an effective technique to widen the coverage and to improve the quality of wireless networks both in terms of performance gain and diversity gain [1]. The simplest cooperation form is three-node relay channel including one source, one relay and one destination, where the relay helps the source to communicate with the destination. In a relay network, the relay estimates the signal from the source and then forwards it to the destination via orthogonal channels. Therefore, the received signal at the destination is the output of two independent channels. It has been shown that the relay network achieves a performance gain and diversity gain compared with non cooperation channel [1]-[2].

There are three main relaying techniques: Decode-andForward (DF), Estimate-and-Forward (EF) and Amplify-andForward (AF). In the first protocol, the relay decodes the received signal and re-encodes it prior transmitting the encoded codeword to the destination [2]. To improve the system coding gain, an interleaver on information bits may be applied to form a distributed turbo code [3]. The second protocol is EF [4], in which the relay estimates the source symbol in order to cooperate with the destination. In AF relaying protocol [5], the relay adjusts the power of the signal received from the source before forwarding it to the destination. All above-mentioned protocols can improve both coding gain and diversity order compared with direct transmission (without relay). When more than one relay is present, to save spectrum efficiency, Relay Selection (RS) [6] is employed to choose the best relay. Most of those papers analyze the relay networks, e.g., in terms of outage probability, in high SNR region.

Recently, there have been increasingly attention on cooperative networks in low and medium SNR regime in various aspects including outage capacity [7] and outage probability [8]. The authors in [9] derived the exact outage probability for arbitrary SNR and asymptotic outage probability of high SNR regime. Note that these results are limited on uncoded systems.

In this paper, we propose a new relaying protocol where the relay forwards only a part of the estimated codeword to the destination to improve the system spectral efficiency. At the destination, a Cooperative Maximal Ratio Combining (C$\mathrm{MRC}$ ) detector is used in order to mitigate error propagation and reduce the system complexity [10] prior to channel decoding. It is well known that the C-MRC achieves full diversity gain and has performance very close to that of a Maximum Likelihood (ML) detector [11]. We name this protocol as partial relaying. Note that the partial relaying was studied in [12] in which a space-time cooperation code was performed. However, no performance analysis was presented in [12]. Over block fading Rayleigh fading channels, we investigate the network performance in terms of bit error rate and diversity gain in low and medium SNR region. The contributions of this paper are twofold: i) First, we compute the upper bound of the BER of the proposed scheme. The upper bound depends on the average SNR, the minimum distance of the channel code and the amount of information the relay forwards. ii) Then, we derive the instantaneous diversity order from the BER upper bound, which allows to analyze the performance in low and medium SNR region. Interestingly, numerical results show that the partial relaying can achieve full diversity gain in a given SNR region (e.g. BER $\leq 10^{-5}$ ) and has $32 \%$ spectrum efficiency improvement as compared with the classical relay network when a strong channel code, i.e., [123 135 157] with minimum distance $d_{H}=15$ is used. Some design and 
optimization aspects are also discussed.

\section{SySTEM MODEL}

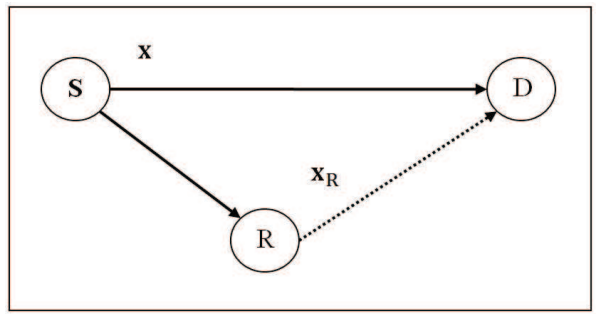

Fig. 1. The three-node relay channel.

The system consists of one source (S) communicating to one destination (D) with the help of one relay (R) as shown in Fig. 1, assuming that all nodes are equipped with single antennas. In what follows, our system is described in terms of orthogonal channels. Therefore, the cooperation is divided in two frames: in the first frame, the source $S$ broadcasts a codeword to the relay and the destination; in the second frame, the relay forwards (part of) the estimated codeword from $\mathrm{S}$ to the destination. All channels are subject to quasistatic block Rayleigh fading plus Additive White Gaussian Noise (AWGN). The relay employs the EF protocol [4]. More specifically, the source $S$ encodes a data message $\mathbf{u}$ of length $K$ into a codeword c of length $N$ via a convolutional code (CC) code of rate of $K / N$. The codeword $\mathbf{c}$ is then BPSK modulated into a signal $\mathbf{x}=\left\{x_{k}\right\}_{k=1}^{N}$ as $x_{k}=2 c_{k}-1$, before being transmitted to the relay and the destination. The received signals at the relay and the destination are modeled as follows:

$$
\begin{aligned}
& \mathbf{y}_{S D}=\sqrt{P_{S D}} h_{S D} \mathbf{x}+\mathbf{n}_{S D}, \\
& \mathbf{y}_{S R}=\sqrt{P_{S R}} h_{S R} \mathbf{x}+\mathbf{n}_{S R},
\end{aligned}
$$

where $P_{X Y}=P_{0} l_{X Y}^{-\eta}$ with $X \in\{S, R\}, Y \in\{R, D\}$ is the power of the received signal at node $\mathrm{Y}$ from node $\mathrm{X}, P_{0}$ is the average transmitted power per symbol, $l_{X Y}$ is the normalized distance from node $\mathrm{X}$ to node $\mathrm{Y}\left(l_{S D}=1\right)$ and $\eta$ is the path loss factor; the channel coefficient $h_{X Y}$ is a complex Gaussian random variable with zero mean and unit variance, i.e. $\mathbb{E}\left\{\left|h_{X Y}\right|^{2}\right\}=1$, and is mutually independent among different $\mathrm{X}-\mathrm{Y}$ channels; $\mathbf{n}_{X Y}$ is a noise vector whose components are Gaussian random variables with zero mean and variance $\sigma^{2}$. For further processing, define $\gamma_{X Y}=P_{X Y}\left|h_{X Y}\right|^{2} / \sigma^{2}$ and $\bar{\gamma}_{X Y}=P_{X Y} / \sigma^{2}$ as the instantaneous and average SNRs of channel X-Y, respectively.

At the end of the first frame, the relay: i) estimates $M=$ $\delta N, 0 \leq \delta \leq 1$ data symbols to form an estimated (punctured) codeword $\hat{\mathbf{c}}_{R}=\left\{\hat{c}_{R, l}\right\}_{l=1}^{M}$. Define $\Theta=\left\{k_{1}, k_{2}, \ldots, k_{M}\right\}$ as the set of data indexes which are retransmitted by the relay, $1 \leq\left\{k_{l}\right\}_{l=1}^{M} \leq N$. The data symbols at the relay are estimated by a Maximum Likelihood (ML) detector: $\hat{c}_{R, l}=$ $\arg \min _{c_{k_{l}} \in\{0,1\}}\left\{\left|y_{S R, k_{l}}-\sqrt{P_{S R}} h_{S R} x_{k_{l}}\right|^{2}\right\}$; ii) modulates $\left\{\hat{c}_{R, l}\right\}_{l=1}^{M}$ into signal symbols $\hat{x}_{R, l}=2 \hat{c}_{R, l}-1$; iii) forwards $\hat{\mathbf{x}}_{R}$ and the index set $\Theta$ to the destination. Unlike [13], which only forwards estimated symbols taking values above a certain threshold, we always forward the estimated symbols. The received signal at the destination from the relay is given by

$$
\mathbf{y}_{R D}=\sqrt{P_{R D}} h_{R D} \hat{\mathbf{x}}_{R}+\mathbf{n}_{R D}
$$

For a fair comparison, the total power at the source and the relay in one cooperative phase is constrained by $P_{t o t}$. Then the symbol power depends on $\delta: P_{0}(\delta)=P_{\text {tot }} /((1+\delta) N)$. Obviously, $\delta=0$ and $\delta=1$ stand for a non-cooperative scheme and conventional relay channel [1], respectively.

After receiving two frames along with the channel state information (CSI) of the S-R channel ${ }^{1}$, the destination decodes in two steps: i) apply the C-MRC detector, ii) then, perform channel decoding. Denote the correct relayed signal as $\mathbf{x}_{R}=\left\{x_{R, k}\right\}_{k=1}^{M}, x_{R, k}=x_{k}, k \in \Theta$. The C-MRC detector operates as follows: $\hat{c}_{k}=\arg \min _{c_{k} \in\{0,1\}}\left\{\mathbb{M}\left(x_{k}\right)\right\}$. The metric $\mathbb{M}\left(x_{k}\right)$ of symbol $x_{k}$ is computed as follows:

$$
\mathbb{M}\left(x_{k}\right)=\left\{\begin{array}{ll}
\left|y_{S D, k}-\sqrt{P_{S D}} h_{S D} x_{k}\right|^{2} & \text { if } k \notin \Theta, \\
\left|y_{S D, k}-\sqrt{P_{S D}} h_{S D} x_{k}\right|^{2} & \\
+\lambda_{R}\left|y_{R D, k}-\sqrt{P_{R D}} h_{R D} x_{R, k}\right|^{2} & \text { if } k \in \Theta
\end{array},\right.
$$

where $\lambda_{R} \triangleq \min \left(\gamma_{S R}, \gamma_{R D}\right) / \gamma_{R D}$ is the factor of C-MRC detector which takes into account possible error of relayed signal [10]. The C-MRC detector then computes the loglikelihood ratio (LLR) of the coded bits $\left\{\hat{c}_{k}\right\}_{k=1}^{N}$ and sends them to the channel decoder which consists in a BCJR algorithm [14] which provides the transmitted data.

\section{Performance AnAlysis}

\section{A. BER Analysis}

The received signal at the destination is the output of block fading channels with 2 blocks [15]: the first block consists of $M$ symbols which are transmitted from the source and the relay, and the second block consists of $N-M$ symbols which propagate only from the source to the destination. Employing the BCJR algorithm, the bit error rate (BER) of the convolutional code is upper bounded as follows [16, eq. (3.175)]:

$$
\mathrm{P}_{e} \leq \sum_{d=d_{H}}^{\infty} w[d] \mathrm{P}_{\mathrm{u}}[d]
$$

where $\mathrm{P}_{\mathrm{u}}[d]$ is the unconditional error probability (UEP) of receiving a codeword with Hamming weight $d$, assuming that the all-zero codeword was transmitted, $d_{H}$ is the minimum distance of the code and $w[d]$ is total number of non-zero input bits according to the codeword with weight $d$. Note that $w[d]$ is computed directly from the code structure. The UEP $\mathrm{P}_{\mathrm{u}}[d]$ is the expectation over the instantaneous SNRs $\left\{\gamma_{S D}, \gamma_{S R}, \gamma_{R D}\right\}$ of the conditional error probability (CEP) as follows:

$$
\mathrm{P}_{\mathrm{u}}[d] \triangleq \mathbb{E}_{\gamma_{S D}, \gamma_{S R}, \gamma_{R D}}\left\{\mathrm{P}_{\mathrm{c}}[d]\right\}
$$

\footnotetext{
${ }^{1}$ The CSI of S-R channel is assumed to be available at the destination via the low-rate feedback.
} 
The CEP depends on the instantaneous SNRs $\gamma_{S D}, \gamma_{S R}, \gamma_{R D}$ and the distribution of the $d$ weights over the 2 blocks. Denoting by $d_{1}$ and $d_{2}$ the weights of block 1 and block 2 , respectively, we have $d_{1}+d_{2}=d$. The weights are distributed over the blocks according to the weight pattern $\mathbf{W}=\left\{d_{1}, d_{2}\right\}$. Then the CEP is computed by averaging over all distributions of the pattern $\mathbf{W}$

$$
\mathrm{P}_{\mathrm{c}}[d]=\sum_{\mathbf{W}} \mathrm{P}_{\mathrm{c}}[d \mid \mathbf{W}] p[\mathbf{W}] .
$$

The probability of the weight pattern $\mathbf{W}$ is computed by combinatorics computation as $p[\mathbf{W}]=\frac{\mathcal{C}_{d_{1}}^{N-M} \times \mathcal{C}_{d_{2}}^{M}}{\mathcal{C}_{d}^{N}}$, where $\mathcal{C}_{k}^{n}=\frac{n !}{k !(n-k) !}$ denotes the binomial coefficients. Substituting (7) into (6), we have

$$
\mathrm{P}_{\mathrm{u}}[d]=\sum_{\mathbf{W}} \mathrm{P}_{\mathrm{u}}[d \mid \mathbf{W}] p[\mathbf{W}]
$$

where $\mathrm{P}_{\mathrm{u}}[d \mid \mathbf{W}] \triangleq \mathbb{E}\left\{\mathrm{P}_{\mathrm{c}}[d \mid \mathbf{W}]\right\}$. The CEP $\mathrm{P}_{\mathrm{c}}[d \mid \mathbf{W}]$ is equal to the probability of receiving the codeword $\widetilde{\mathrm{x}}$ when the allzero codeword $\mathbf{x}$ was transmitted [16], conditioned on that the Hamming distance between two vectors $\mathcal{H}(\mathbf{x}, \widetilde{\mathbf{x}})=d$. It is given by

$$
\begin{aligned}
\mathrm{P}_{\mathrm{c}}[d \mid \mathbf{W}]= & \operatorname{Pr}\left\{\mathbf{x} \rightarrow \widetilde{\mathbf{x}} \mid \mathbf{W}, \mathcal{H}(\mathbf{x}, \widetilde{\mathbf{x}})=d, \gamma_{S R}, \gamma_{S D}, \gamma_{R D}\right\} \\
= & \operatorname{Pr}\{\mathbb{M}(\mathbf{x})-\mathbb{M}(\widetilde{\mathbf{x}})>0 \mid \mathbf{W} \\
& \left.\mathcal{H}(\mathbf{x}, \widetilde{\mathbf{x}})=d, \gamma_{S R}, \gamma_{S D}, \gamma_{R D}\right\}
\end{aligned}
$$

The difference between two metrics can be computed from (4) as

$$
\begin{aligned}
\mathbb{M}(\mathbf{x})-\mathbb{M}(\widetilde{\mathbf{x}})= & \sum_{k=1}^{d} \frac{2 \sqrt{P_{S D}} h_{S D} y_{S D, k}\left(\widetilde{x}_{k}-x_{k}\right)}{\sigma^{2}} \\
& +\lambda_{R} \sum_{l=1}^{d_{1}} \frac{2 \sqrt{P_{R D}} h_{R D} y_{R D, l}\left(\widetilde{x}_{R, l}-x_{R, l}\right)}{\sigma^{2}}
\end{aligned}
$$

where (10) holds because of i) given $\mathcal{H}(\mathbf{x}, \widetilde{\mathbf{x}})=d$, there is only $d$ different symbols between $\mathbf{x}$ and $\widetilde{\mathbf{x}}$; ii) given the weight pattern $\mathbf{W}=\left\{d_{1}, d_{2}\right\}$, there is $d_{1}$ different symbols between $\mathbf{x}_{R}$ and $\widetilde{\mathbf{x}}_{R}$; and iii) without loss of generality, it is assumed that the first $d$ symbols of $\widetilde{\mathbf{x}}$ differ from that of $\mathbf{x}$, e.g. $\mid \widetilde{x}_{k}-$ $x_{k} \mid \neq 0$ for $1 \leq k \leq d$ and $\left|\widetilde{x}_{k}-x_{k}\right|=0$ for $d<k \leq N$, and the first $d_{1}$ symbols of $\mathbf{x}_{R}$ differ from that of $\widetilde{\mathbf{x}}_{R}$, e.g. $\left|\widetilde{x}_{R, l}-x_{R, l}\right| \neq 0$ for $1 \leq l \leq d_{1}$ and $\left|\widetilde{x}_{R, l}-x_{R, l}\right|=0$ for $d_{1}<l \leq M$.

Denote $\mathbf{x}_{R}^{d_{1}}=\left\{x_{R, k}\right\}_{k=1}^{d_{1}}$ as the first $d_{1}$ symbols of $\mathbf{x}_{R}$. Taking into account possible decoding errors at the relay, the probability in (9) can be re-written as follows:

$$
\begin{aligned}
\mathrm{P}_{\mathrm{c}}[d \mid \mathbf{W}]= & \operatorname{Pr}\left\{\hat{\mathbf{x}}_{R}^{d_{1}}=\mathbf{x}_{R}^{d_{1}}\right\} \operatorname{Pr}\left\{\mathbb{M}(\mathbf{x})-\mathbb{M}(\widetilde{\mathbf{x}})>0 \mid \mathbf{x}_{R}\right\} \\
& +\operatorname{Pr}\left\{\hat{\mathbf{x}}_{R}^{d_{1}} \neq \mathbf{x}_{R}^{d_{1}}\right\} \operatorname{Pr}\left\{\mathbb{M}(\mathbf{x})-\mathbb{M}(\widetilde{\mathbf{x}})>0 \mid \hat{\mathbf{x}}_{R}\right\} .
\end{aligned}
$$

It is assumed that if an error occurs in $\hat{\mathbf{x}}_{R}^{d_{1}}$, there is only one symbol error. This approximation is valid for block fading and a small value of $d_{1}$, leading to $\operatorname{Pr}\left\{\hat{\mathbf{x}}_{R}^{d_{1}} \neq \mathbf{x}_{R}^{d_{1}}\right\}=$
$Q\left(\sqrt{\gamma_{S R}}\right)$ and $\operatorname{Pr}\left\{\hat{\mathbf{x}}_{R}^{d_{1}}=\mathbf{x}_{R}^{d_{1}}\right\}=1-Q\left(\sqrt{\gamma_{S R}}\right)$, with $Q(x)=\frac{1}{\sqrt{2 \pi}} \int_{x}^{\infty} e^{-t^{2} / 2} d t$. Then (11) becomes

$$
\begin{aligned}
\mathrm{P}_{\mathrm{c}}[d \mid \mathbf{W}]= & \left(1-Q\left(\sqrt{\gamma_{S R}}\right)\right) \operatorname{Pr}\left\{\mathbb{M}(\mathbf{x})-\mathbb{M}(\widetilde{\mathbf{x}})>0 \mid \mathbf{x}_{R}\right\} \\
& +Q\left(\sqrt{\gamma_{S R}}\right) \operatorname{Pr}\left\{\mathbb{M}(\mathbf{x})-\mathbb{M}(\widetilde{\mathbf{x}})>0 \mid \hat{\mathbf{x}}_{R}\right\} .
\end{aligned}
$$

Denote $\overline{\mathbf{x}}_{R} \in\left\{\mathbf{x}_{R}, \hat{\mathbf{x}}_{R}\right\}$, then the probability in (12) can be expressed as follows:

$$
\begin{aligned}
\operatorname{Pr}\{\mathbb{M}(\mathbf{x}) & \left.-\mathbb{M}(\widetilde{\mathbf{x}})>0 \mid \overline{\mathbf{x}}_{R}\right\} \\
= & \operatorname{Pr}\left\{\Delta_{S}(\mathbf{x}, \widetilde{\mathbf{x}})+\lambda_{R} \Delta_{R}\left(\overline{\mathbf{x}}_{R}, \mathbf{x}_{R}, \widetilde{\mathbf{x}}_{R}\right)<0\right\},
\end{aligned}
$$

where

$$
\begin{aligned}
\Delta_{S}(\mathbf{x}, \widetilde{\mathbf{x}}) & =\sum_{k=1}^{d} \frac{2 \sqrt{P_{S D}} h_{S D} y_{S D, k}\left(x_{k}-\widetilde{x}_{k}\right)}{\sigma^{2}} \\
\Delta_{R}\left(\overline{\mathbf{x}}_{R}, \mathbf{x}_{R}, \widetilde{\mathbf{x}}_{R}\right) & =\sum_{l=1}^{d_{1}} \frac{2 \sqrt{P_{R D}} h_{R D} y_{R D, l}\left(x_{R, l}-\widetilde{x}_{R, l}\right)}{\sigma^{2}} .
\end{aligned}
$$

Since the all-zero codeword is transmitted, e.g, $x_{k}=-1$, then we have $A_{\widetilde{\mathbf{x}} \mathbf{x}}=\widetilde{x}_{k}-x_{k}$ for all $1 \leq k \leq d$ and $A_{\widetilde{\mathbf{x}}_{R} \mathbf{x}_{R}}=\widetilde{x}_{R, k}-x_{R, k}$ for all $1 \leq k \leq d_{1}$. Denote the normalized noise variables $\bar{n}_{S D} \triangleq \sum_{k=1}^{d} n_{S D, k} /(\sigma \sqrt{d})$ and $\bar{n}_{R D} \triangleq \sum_{l=1}^{d_{1}} n_{R D, l} /\left(\sigma \sqrt{d_{1}}\right)$. It is easy to show that $\bar{n}_{S D}, \bar{n}_{R D} \sim \mathcal{C N}(0,1)$. Then $\Delta_{S}(\mathbf{x}, \widetilde{\mathbf{x}})$ and $\Delta_{R}\left(\overline{\mathbf{x}}_{R}, \mathbf{x}_{R}, \widetilde{\mathbf{x}}_{R}\right)$ can be rewritten as

$$
\begin{aligned}
\Delta_{S}(\mathbf{x}, \widetilde{\mathbf{x}})= & \left|\sqrt{d \gamma_{S D}} A_{\widetilde{\mathbf{x}} \mathbf{x}}-\bar{n}_{S D}\right|^{2}-\left|\bar{n}_{S D}\right|^{2}(14) \\
\Delta_{R}\left(\overline{\mathbf{x}}_{R}, \mathbf{x}, \widetilde{\mathbf{x}}\right)= & \left|\sqrt{d_{1} \gamma_{R D}} A_{\widetilde{\mathbf{x}}_{R} \overline{\mathbf{x}}_{R}}-\bar{n}_{R D}\right|^{2} \\
& -\left|\sqrt{d_{1} \gamma_{R D}} A_{\overline{\mathbf{x}}_{R} \mathbf{x}_{R}}-\bar{n}_{R D}\right|^{2}
\end{aligned}
$$

The conditional error probability $\mathrm{P}_{c}[d \mid \mathbf{W}]$ depends on the distribution of the pattern $\mathbf{W}$ and is computed as follows:

Theorem 1. Denote $D_{1}=\left\{d_{1}=0, d_{2}=d\right\}$ as the pattern such that all $d$ weights are located in block 2 (the one without help of the relay). The UEP $\mathrm{P}_{\mathrm{u}}\left[d \mid D_{1}\right] \triangleq$ $\mathbb{E}_{\gamma_{S D}, \gamma_{S R}, \gamma_{R D}}\left\{\mathrm{P}_{\mathrm{c}}\left[d \mid D_{1}\right]\right\}$ is upper bounded by

$$
\mathrm{P}_{\mathrm{u}}\left[d \mid D_{1}\right] \leq \frac{1}{4 d \bar{\gamma}_{S D}}
$$

where $\bar{\gamma}_{S D}$ is the expectation of $\gamma_{S D}$.

Proof. Given the weight pattern $D_{1}=\{0, d\}$, the metrics do not involve any symbol which has been forwarded by the relay. Therefore, $\mathbb{M}(\mathbf{x})-\mathbb{M}(\widetilde{\mathbf{x}})=\Delta_{S}(\mathbf{x}, \widetilde{\mathbf{x}})=$ $\left|\sqrt{d \gamma_{S D}} A_{\widetilde{\mathbf{x}} \mathbf{x}}-\bar{n}_{S D}\right|^{2}-\left|\bar{n}_{S D}\right|^{2}$. From (11), the CPEP $\mathrm{P}_{\mathrm{c}}\left[d \mid D_{1}\right]=\operatorname{Pr}\left\{\Delta_{S}(\mathbf{x}, \widetilde{\mathbf{x}})<0 \mid \gamma_{S R}, \gamma_{S D}, \gamma_{R D}\right\}$. With the help of [17], the UEP is integrated over all instantaneous channel gains of CEP as follows:

$$
\mathrm{P}_{\mathrm{u}}\left[d \mid D_{1}\right]=\mathbb{E}_{\gamma_{S D}}\left\{Q\left(\sqrt{d \gamma_{S D}}\right)\right\} \leq \frac{1}{4 d \bar{\gamma}_{S D}}
$$

Theorem 2. Consider the weight pattern $\mathbf{W}=\left\{d_{1}>0, d_{2}=\right.$ $\left.d-d_{1}\right\} \neq D_{1}$. The UEP $\mathrm{P}_{\mathrm{u}}[d \mid \mathbf{W}] \triangleq \mathbb{E}_{\gamma_{S D}, \gamma_{S R}, \gamma_{R D}}\left\{\mathrm{P}_{\mathrm{c}}[d \mid \mathbf{W}]\right\}$ is upper bounded by

$$
\mathrm{P}_{\mathrm{u}}[d \mid \mathbf{W}] \leq \frac{\alpha}{d d_{1} \bar{\gamma}_{S D} \bar{\gamma}_{R D}}+\frac{\beta}{d d_{1} \bar{\gamma}_{S D} \bar{\gamma}_{S R}}, \forall \mathbf{W} \neq D_{1}
$$


where $\alpha=3 / 16$ and $\beta=(45+\sqrt{5}) / 160$.

Proof. Due to space limitation, the proof which follows the same method as in [11, Sec. 3] is omitted.

The UEP $\mathrm{P}_{\mathrm{u}}[d]$ is given by Theorem 1 and Theorem 2 as follows:

$$
\begin{aligned}
\mathrm{P}_{\mathrm{u}}[d] \leq & \frac{1}{4 d \bar{\gamma}_{S D}} p\left(D_{1}\right) \\
& +\left(\frac{\alpha}{d \bar{\gamma}_{S D}^{2} g_{R D}}+\frac{\beta}{d \bar{\gamma}_{S D}^{2} g_{S R}}\right) \sum_{\mathbf{W} \neq D_{1}} \frac{p(\mathbf{W})}{d_{1}}
\end{aligned}
$$

with $g_{S R}=\bar{\gamma}_{S R} / \bar{\gamma}_{S D}, g_{R D}=\bar{\gamma}_{R D} / \bar{\gamma}_{S D}$.

From (19) we can see that the BER of the proposed scheme is a sum of two terms: one has diversity order 1 with weight $p\left[D_{1}\right] /(4 d)$ and the other has diversity order 2 . The overall performance (BER and diversity gain) depends on the ratio between the weights of both terms. The probability of the diversity 1 term is given by $p\left[D_{1}\right]=\prod_{k=0}^{d-1} \frac{N(1-\delta)-k}{N-k}$. In practice, the codeword length $N$ is usually much larger than $d$ (for example $d=7$ for the RSC [ [ $\left.\begin{array}{lll}5 & 7 & 5\end{array}\right]$ code and $N$ is usually several hundreds of symbols), then $p\left[D_{1}\right]$ can be wellapproximated as

$$
p\left[D_{1}\right] \simeq(1-\delta)^{d} .
$$

Depending on $d$ and $\delta$, the contribution of the diversity order 1 term changes compared with that of the diversity order 2 . The larger the values of $\delta$ and $d$ are, the smaller the contribution of diversity order 1 factor is, and vice versa.

\section{B. Diversity Analysis}

The classical definition of diversity order is defined as negative exponent of the average $\mathrm{BER} \mathrm{P}_{e}$ in log-log scale in the infinite $\mathrm{SNR}$ region [18] as $\zeta \triangleq-\lim _{\gamma \rightarrow \infty} \frac{\log \left[\mathrm{P}_{e}(\gamma)\right]}{\log (\gamma)}$, where $\gamma$ is the average SNR. Visually, the diversity order is slope of the plot of BER as a function of average SNR when SNR tends to infinity. In this paper, we are interested in the diversity order in low and medium SNR region. We define diversity order at a certain average $\mathrm{SNR} \gamma$ as follows:

$$
\zeta(\gamma) \triangleq-\lim _{\Delta \rightarrow 1} \frac{\log \left[\mathrm{P}_{2}(\Delta \gamma)\right]-\log \left[\mathrm{P}_{e}(\gamma)\right]}{\log (\Delta \gamma)-\log (\gamma)}
$$

which obviously matches the classical definition of diversity when the SNR tends to infinity. Because the diversity order depends on the average SNR, we refer to $\zeta(\gamma)$ as instantaneous diversity order. The key idea behind the definition is that it allows to study the behavior of the system at any SNR values. This is crucial to practical systems since they usually operate at finite SNR region. Generally speaking, the instantaneous diversity order is the slope when we plot $\mathrm{P}_{e}(\gamma)$ as a function of $\gamma$ in log-log scale. Mathematically speaking, the instantaneous diversity at $\gamma_{0}$ is the derivative of $\log \left[\mathrm{P}_{e}(\gamma)\right]$ in $\log$ scale as

$$
\begin{aligned}
\zeta\left(\gamma_{0}\right) & =-\left.\frac{\partial}{\partial \log \gamma} \log \left[\mathrm{P}_{e}(\gamma)\right]\right|_{\gamma=\gamma_{0}} \\
& =-\left.\gamma \frac{\partial}{\partial \gamma} \ln \left[\mathrm{P}_{e}(\gamma)\right]\right|_{\gamma=\gamma_{0}} .
\end{aligned}
$$

Using instantaneous diversity definition, the diversity order of the proposed network in low and medium SNR region is given by Theorem 3 .

Theorem 3. The instantaneous diversity order of $\mathrm{P}_{u}[d]$ at average SNR $\gamma_{0}$ of the proposed system is given by

$$
\zeta\left(\gamma_{0}\right)=1+\frac{B}{B+A \gamma_{0}}
$$

where $A=(1-\delta)^{d}$ and

$$
B=\left(\frac{3}{4 g_{R D}}+\frac{45+\sqrt{5}}{40 g_{S R}}\right) \sum_{\mathbf{W} \neq D_{1}} \frac{p(\mathbf{W})}{d_{1}} .
$$

Proof. Define $\mathrm{F}(\gamma)=\log \left(\mathrm{P}_{u}[d]\right)=\log \left(A \gamma^{-1} /(4 d)+\right.$ $\left.B \gamma^{-2} /(4 d)\right)$, the derivative of $\mathrm{F}(\gamma)$ is given as follows:

$$
\mathrm{F}^{\prime}(\gamma)=-\gamma^{-1}\left(1+\frac{B}{B+A \gamma}\right)
$$

Substituting $\mathrm{F}^{\prime}(\gamma)$ into (23) and setting $\gamma=\gamma_{0}$ we have

$$
\zeta\left(\gamma_{0}\right)=1+\frac{B}{B+(1-\delta)^{d} \gamma_{0}} .
$$

Theorem 3 states that the instantaneous diversity order is a function of $\delta, d$ and $\gamma$. If $d$ and $\delta$ are large enough such as (1$\delta)^{d} \gamma_{0} \ll B$, the proposed scheme can achieve a diversity order of 2 in $\left[0, \gamma_{0}\right]$. We note from (5) that since the system BER is proportional to the UEP $\mathbf{P}_{\mathbf{u}}[d]$ via the input weight $w[d]$ which is fixed for given channel code, the instantaneous SNR of the system is given in Theorem 3. Especially, i) if the relay stays silent, e.g., $\delta=0$, then $B=0$ and the system diversity order is one; ii) if the relay forwards the entire codeword, e.g., $\delta=1$, then $\zeta(\gamma)=2 \forall \gamma$. In addition, the diversity of partial relaying $(0<\delta<1)$ approaches to 1 when the average SNR tends to infinity. However, we note again that our design focuses on practical systems whose operating SNR region is finite.

\section{NumERICAL AND Simulation RESUlts}

The channel and system setup are as follows: the source transmits a message of 200 bits long, BPSK modulated. The total power $P_{t o t}$ is fixed whatever $\delta$. The relay is located at the middle of the source and the destination, e.g., $l_{S R}=$ $l_{R D}=0.5$ and $\eta=4$. Three channel code with rate $1 / 3$ are examined [19]: i) CC [3 2 2 3] with $d_{H}=5$, ii) CC [5 7 5] with $d_{H}=7$, and iii) CC [123 135 157] with $d_{H}=15$. Fig. 2 shows the upper bound and simulation of the proposed network. The solid curves show the simulation results and the dotted curves show the upper bound in (19). Three first weights in (5) are used to compute the bound ${ }^{2}$. It is shown that the upper bound is quite tight compared to simulation results. Interestingly, the strong code with $\delta=1 / 2$ achieves full diversity gain in the SNR region where BER $\leq 10^{-5}$ and provides the same performance as classical relaying $(\delta=1)$ in this region, while $32 \%$ of spectrum efficiency is saved. Fig. 3

\footnotetext{
${ }^{2}$ While the maximum weight $d$ in (5) can be infinity for Gaussian channels, it is usually limited by several first values for block Rayleigh fading channels
} 

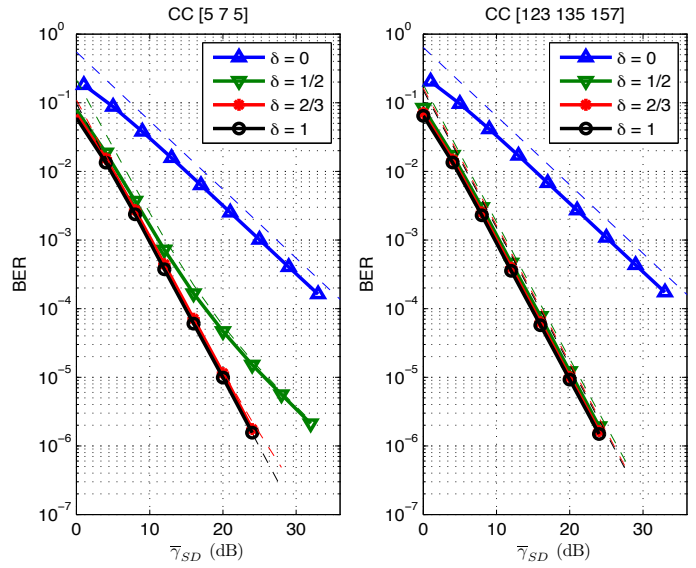

Fig. 2. Effect of $\delta$ on performance of the proposed network for different channel codes. Solid marked cures: simulation results, dotted curves: the bound.
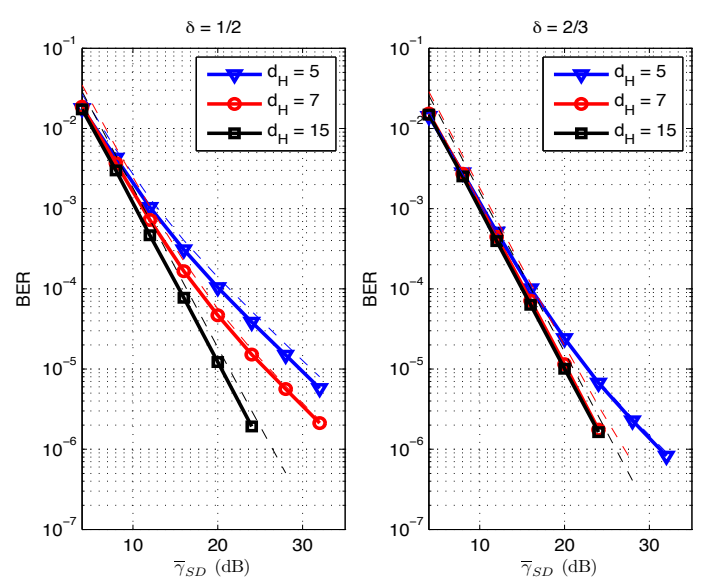

Fig. 3. Effect of minimum distance $d_{H}$ on performance of the proposed network for different $\delta$. Solid marked cures: simulation results, dotted curves: the bound.

shows the impact of the choice of the channel code on the diversity order. When the relay cooperates $\delta=2 / 3$ codeword ( $20 \%$ spectrum efficiency saving), both the strong and medium code ([ $\left.\left.\begin{array}{lll}5 & 7 & 5\end{array}\right]\right)$ provide full diversity in the SNR region of interest.

\section{Discussion AND CONCLUSION}

Theorem 3 provides the connections between instantaneous diversity order, the channel code and the amount of relayed information. This relation provides a criterion to design the relay network in order to, for example, achieve full diversity gain in a given SNR region via properly choosing the code and $\delta$. In practical networks, while the channel code is usually chosen among a finite set, the relaying factor $\delta$ is more flexible. Another aspect that can be considered is the position of the relay. This can be done by minimizing the upper bound of
BER. Note that we study in this paper the quasi-static block Rayleigh fading, by our method can be extended to general block fading channels.

In conclusion, we proposed a new channel-coded cooperative relay network in which the relay can retransmit the whole or only part of the estimated codeword in order to save spectrum efficiency. We derived a so-called instantaneous diversity order in low and medium SNR region. The instantaneous diversity provides a good understanding of the system. It was also shown that with a strong code, the proposed system can achieve full diversity gain in low and medium SNR region, while saving some spectrum efficiency when compared to the classical relay network.

\section{REFERENCES}

[1] A. Sendonaris, E. Erkip, and B. Aazhang, "User cooperation diversity Part II: Implementation aspects and performance analysis," IEEE Trans. Commun., vol. 51, no. 11, pp. 1939-1948, Nov. 2003.

[2] E. V. Meulen, "Three-terminal communication channels," Advances in Applied Probability, vol. 3, no. 1, pp. 120-154, 1971.

[3] B. Zhao and M. C. Valenti, "Distributed turbo coded diversity for relay channel," Electron. Lett., vol. 39, no. 10, pp. 786-787, May 2003.

[4] T. Cover and A. E. Gamal, "Capacity theorems for the relay channel," IEEE Trans. Inf. Theory, vol. 25, no. 5, pp. 572-584, May 1979.

[5] G. Kramer, M. Gastpar, and P. Gupta, "Cooperative strategies and capacity theorems for relay networks," IEEE Trans. Inf. Theory, vol. 51, no. 9, pp. 3037-3063, Sep. 2005.

[6] A. Bletsas, A. Khisti, D. P. Reed, and A. Lippman, "A simple cooperative diversity method based on network path selection," IEEE J. Sel. Areas Commun., vol. 24, no. 3, pp. 659-672, Mar. 2006.

[7] A. S. Avestimehr and D. N. C. Tse, "Outage capacity of the fading relay channel in the low-SNR regime," IEEE Trans. Inf. Theory, vol. 53, no. 4, pp. 1401-1415, Apr. 2007.

[8] E. Beres and R. Adve, "Outage probability of selection cooperation in the low to medium SNR regime," IEEE Commun. Lett., vol. 11, no. 7, pp. 589-597, Jul. 2007.

[9] K. Tourki, H. Yang, and M. Alouini, "Accurate outage analysis of incremental decode-and-forward opportunistic relaying," IEEE Trans. Wireless Commun., vol. 10, no. 4, pp. 1021 - 1025, Apr. 2011.

[10] T. Wang, A. Cano, G. B. Giannakis, and J. N. Laneman, "Highperformance cooperative demodulation with decode-and-forward relays," IEEE Trans. Commun., vol. 55, no. 7, pp. 1427-1438, Jul. 2007.

[11] A. Nasri, R. Schober, and M. Uysal, "Performance and optimization of network-coded cooperative diversity systems," IEEE Trans. Commun., 2013, to appear.

[12] M. Janani, A. Hedayat, T. E. Hunter, and A. Nosratinia, "Coded cooperation in wireless communications: space-time transmission and iterative decoding," IEEE Trans. Signal Process., vol. 52, no. 2, pp. 362-371, Feb. 2004.

[13] N. Sinh Le Hong, A. Ghrayeb, G. Al-Habian, and M. Hasna, "Mitigating error propagation in two-way relay channels with network coding," IEEE Trans. Wireless Commun., vol. 9, no. 11, pp. 3380-3390, Nov. 2010.

[14] L. Bahl, J. Cocke, F. Jelinek, and J. Raviv, "Optimal decoding of linear codes for minimizing symbol error rate (corresp.)," IEEE Trans. Inf. Theory, vol. 20, no. 2, pp. 284-287, Feb. 1974.

[15] R. Knopp and P. A. Humblet, "On coding for block fading channels," IEEE Trans. Inf. Theory, vol. 46, no. 1, pp. 189-205, Jan. 2000.

[16] A. Glavieux, Channel Coding in Communication Networks: From Theory to Turbo Codes. Wiley\& Sons Ltd, 2007.

[17] M. K. Simon and M.-S. Alouini, Digital communication over fading channels, 2nd ed. Hoboken, N.J.: John Wiley \& Sons, 2005.

[18] M. Uysal, "Diversity analysis of space-time coding in cascaded Rayleigh fading channels," IEEE Commun. Lett., vol. 10, no. 3, pp. 165-167, Mar. 2006.

[19] S. Benedetto, R. Garello, and G. Montorsi, "A search for good convolutional codes to be used in the construction of turbo codes," IEEE Trans. Commun., vol. 46, no. 9, pp. 1101-1105, Sep. 1998. 